\title{
I Aprendizaje y enseñanza de la historia. Parti- cipación e historia en educación de adultos
}

\author{
Graciela Hernández ${ }^{1}$ \\ Jessica Visotsky²
}

\section{Introducción}

A lo largo de nuestro trabajo de historia oral en educación de adultos hemos puesto interés en reflexionar acerca de la educación y su relación con la cultura, por lo tanto es imprescindible que nos planteemos qué es la cultura y a qué cuestiones teóricas nos lleva esta pregunta. Descartamos las respuestas ingenuas propias del culturalismo antropológico que evaden el conflicto y afirman simplemente "que todos los seres humanos poseen cultura"; porque esta afirmación oculta más de lo que muestra. También descartamos las respuestas desde el humanismo que encontraría carentes de cultura a estos sujetos sociales convertidos en alumnos en la edad adulta.

Desde el marxismo tradicional, la cultura es vista como un elemento "superestructural" de la sociedad. Sin embargo, también algunos teóricos marxistas como Louis Althusser y Antonio Gramsci, buscaron desprenderse del economicismo subyacente a los análisis tradicionales, para mostrar la complejidad de las relaciones entre cultura y economía. Estos planteos llevan a un cuestionamiento de nociones marxistas como ideología, clase social y dominación. Con el estructuralismo y el marxismo de los años setenta las ciencias sociales tuvieron la oportunidad de desprenderse del concepto humanista de "sujeto" como eje de los procesos culturales. Entonces, la cultura se puede estudiar también como el terreno donde tiene lugar la lucha por el poder y donde se cristalizan las formas simbólicas de dominación.

Sin dudas Gramsci es una figura importante en el pensamiento marxista y crecientemente influyente en una amplia variedad de campos. Además

1- CONICET- Universidad Nacional de La Pampa (Desarrolló los puntos 1 y 2).

2- Doctoranda en Historia Universidad Nacional del Sur, Alumna Especialización en Investigación Educativa Escuela Marina Vilte- Universidad Nacional del Comahue (Desarrolló los puntos de 3 a 6). 
de tener un lugar destacado en la lucha revolucionaria es también una figura emblemática por su valoración de la educación de adultos, a la cual consideraba fundamental para lograr una hegemonía revolucionaria.

Gramsci influyó mucho en los intelectuales ingleses. En 1964 se fundó el Centro de Estudios de la Cultura Contemporánea en Birmingham, entre las principales figuras se encontraban: Raymond Williams, Richard Hoggart, Stuart Hall y Edward Thompson. Esta escuela, enrolada en el marxismo, desarrolló una importante tarea extra-académica, trabajando con adultos obreros, en procesos de educación no formal. Le otorgaron un lugar muy importante a la contraposición entre cultura popular y cultura de masas.

En América Latina, el autor más citado por su trayectoria en la educación de adultos es, sin duda, Paulo Freire. Para el pedagogo brasileño, el conocimiento de la cultura de los sujetos que se estaban alfabetizando era un elemento fundamental en este proceso. El "círculo de cultura" es clave en el método freireano y se trata del núcleo del diálogo intersubjetivo que no pretende ser un método de enseñanza sino de aprendizaje.

En este artículo intentaremos reflexionar acerca de la cultura, la historia, y la enseñanza y aprendizaje de la historia a partir de nuestra experiencia en la realización de "Talleres de Historia" en establecimientos de alfabetización de adultos. Como consecuencia de estos planteos analizaremos cómo se articulan las reflexiones con la participación social.

\section{1- Los "Talleres de Historia"}

El ámbito de realización de estos talleres son los sectores populares urbanos con un pasado rural, en la ciudad de Bahía Blanca. Gran parte de esta migración se dio desde Chile y desde la Patagonia argentina, mientras que algunos de estos migrantes partieron de comunidades indígenas y se consideran a sí mismos como mapuche, tanto unos como otros (campesinos e indígenas) aunque con particularidades y reivindicaciones étnicas distintas comparten muchos elementos culturales producto de los procesos socioculturales del área. Hay un número menor de migrantes bolivianos y de otros lugares del país. Siempre predomina el pasado campesino.

Comenzamos a trabajar en escuelas de adultos desde distintas perspectivas. Por un lado, a través de la realización de "Talleres de Historia", en los cuales se abordaba a partir de la oralidad temas vinculados a la historia reciente y a la memoria sobre la cultura y el pasado. Por otro lado, nos dedi- 
camos a los temas vinculados a la alfabetización y al proceso de apropiación de la lecto-escritura y su entorno social y político. Las coincidencias de espacios y de objetivos nos llevaron a plantearnos un trabajo en conjunto en el cual historia oral y alfabetización encontraran puntos en común. A partir de 1999, empezamos a trabajar en una línea de investigación que continúa hasta el presente y que Ilevamos a cabo tanto en Centros de Alfabetización Municipal como en escuelas provinciales de adultos.

Durante ese año realizamos dos "Talleres de Historia" en espacios de alfabetización en dos anexos de una escuela provincial de adultos en un barrio denominado "Villa Rosario" (Hernández y Visotsky 2001). Al año siguiente repetimos esta experiencia pero en un sector diferente de la ciudad, en este caso se trataba de los barrios Vista Alegre y $1^{\circ}$ de Mayo, en Centros de Alfabetización Municipal, al igual que en el 2001 que trabajamos en el Centro de Alfabetización Municipal del Barrio Rivadavia.

Durante el 2003, realizamos el taller que da lugar a este artículo en una escuela provincial de adultos donde concurren -como en todos los casos- mayoritariamente mujeres, la mayor parte de ellas beneficiarias del Plan Jefas y Jefes de Hogar.

En estos talleres hemos dialogado sobre las migraciones, los espacios vividos y transitados, los recuerdos, con valoraciones y datos. Abordamos el problema de alimento y tratamos de realizar una historia que dé cuenta del cambio cultural a través de la comida, como así también del vestido y de la importancia de las prácticas textiles. También trabajamos el tema del universo simbólico expresado a través de relatos de antiguas creencias que aún siguen vigentes y que se van resignificando en la ciudad, generalmente en relación con la participación en iglesias evangélicas pentecostales o en instituciones católicas, especialmente Cáritas. Se realizaban producciones escritas donde se registraban reflexiones acerca de lo trabajado en el taller. Con los testimonios orales transcriptos y seleccionados (sujetos a la evaluación del grupo) realizamos un cuadernillo, el cual se reparte a todos los participantes el día que concluyen los talleres, generalmente en una pequeña despedida a la que se trata de dar un entorno festivo. También tomamos un registro de video y en todos los casos se expone la filmación en el ámbito escolar.

Tratamos de incluir testimonios acerca de las valoraciones de su lugar de origen, las causas para migrar y la forma de trasladarse a Bahía Blanca desde diferentes lugares del país y de Chile. Le dimos un lugar especial a los relatos de los traslados a pie a través de la Cordillera. También incluimos la 
perspectiva de género para registrar los testimonios de varones y mujeres, sus particularidades con los valores y datos que aparecen en los relatos. ${ }^{3}$

Pensamos a este trabajo en talleres como en una estrategia metodológica propia de la historia oral y consideramos que, desde la historia oral, se puede hacer visible la cultura de las clases subalternas (sectores populares urbanos) y su relación con la historia que se enseña en el ámbito escolar. Generalmente, el tema de las condiciones de la partida del lugar de origen y de la llegada es uno de los últimos en ser tratados, ya que es posible hacerlo sólo cuando el grupo del taller se consolida y es factible abordar desde el recuerdo situaciones que han sido dolorosas, llenas de incertidumbres, de vicisitudes, de angustias y a la vez de esperanzas. ${ }^{4}$

\section{a- Los "Talleres de Historia" realizados en la Escuela No 705. Anexo San Dionisio}

Este anexo de la escuela provincial primaria $N^{0} 705$ se encuentra en el área de la Delegación Noroeste de la ciudad, la cual es reconocida por haber recibido una fuerte migración de Chile y de las provincias patagónicas. En la periferia de esta zona se encuentran asentamientos que siempre han complementado sus actividades laborales con la recolección y venta de metales, papel, vidrio y otros materiales, pero en la última década, con la pérdida del empleo, el "cartoneo" se ha convertido en la principal fuente de ingresos, junto con los planes sociales.

Las instituciones de alfabetización siempre están cruzadas de una u otra forma por las políticas públicas para enfrentar a la pobreza, pero éstas han ido sufriendo modificaciones en los últimos años. En primer término, queremos señalar que los alumnos de las escuelas primarias de adultos no recibían ningún tipo de ayuda social estatal, mientras que los que concurrían a los centros municipales recibían un "bolsón de comida", se trataba de ali-

3- El tema de los relatos de mujeres migrantes fue analizado especialmente en el trabajo: "Una mirada diacrónica a las concepciones de familia en sectores populares urbanos con fuerte migración rural del interior de país y de Chile. Desde fines de la década del 50' hasta el presente, en la ciudad de Bahía Blanca.”, presentado en: VII Jornadas de Historia de las Mujeres. II Congreso Iberoamericano de Estudios de Género, Salta, Julio de 2003.

4- El tema de las relaciones entre los talleres de historia y la historia oral fue abordado en el trabajo: "Historia oral y cultura popular. La historia narrada desde las escuelas de alfabetización de adultos." VI Encuentro Nacional de Historia Oral "Historia Oral, una mirada desde el siglo XXI". 
mentos secos que eran repartidos por las propias maestras. Luego el municipio retiró esta ayuda social y se centralizó el reparto de alimentos.

Durante el transcurso del año 2001, algunas de las alumnas lograban que no se les exigiera otra contraprestación como recepción de los planes sociales que asistir a la escuela o centros de alfabetización; este logro dependía de las trabajadoras sociales y de "voluntades" individuales. Lo que fue una excepción se fue convirtiendo en norma, cuando realizamos el taller de historia en el Anexo San Dionisio de la Escuela 705, pudimos comprobar que las alumnas adultas mayores que concurrían para concluir el ciclo de EGB recibían este plan.

La condición de alumnas -los alumnos varones no tenían continuidad y prácticamente no participaron de los talleres- y "beneficiarias" de los planes que asisten a la escuela como contraprestación al beneficio recibido, introduce una nueva variable para comprender el complejo proceso de la alfabetización de adultos. El analfabetismo produce exclusiones y la superación de esta limitación debería ser un factor de inclusión y participación, en la cual las reflexiones sobre la historia y la propia identidad tendrían que tener un lugar destacado; sin embargo la realidad que se nos presenta es bastante más compleja y no hay relaciones preestablecidas de causa-efecto.

En nuestro trabajo en talleres durante el 2003 le dimos mucha importancia a las reflexiones acerca de la participación y los reclamos a las autoridades producidos durante el acto de celebración del 25 de Mayo.

\section{b- El acto en conmemoración del 25 de Mayo}

Comenzamos a trabajar en estos talleres en el mes de abril de 2003. Debido a que la frecuencia era de encuentros quincenales, no teníamos afianzada nuestra relación con el grupo para el mes de mayo, sin embargo las maestras del establecimiento nos propusieron que colaboráramos con el acto del 25 de mayo. Ambas docentes planearon el acto de la siguiente manera: 1. Lectura de un poema por parte de una alumna. 2. Realización de la representación de un cabildo abierto. 3. Festejo con empanadas que se cocinarían en la cocina del establecimiento.

Se nos asignó la coordinación del "cabildo" en el cual las "cabildantas" expresarían cuáles eran los problemas de su barrio, sus preocupaciones, sus propuestas. Accedimos gustosamente a esta solicitud y actuamos como moderadoras de este cabildo. Las propias docentes y las alumnas armaron la 
escenografía y enfatizaron en la importancia de anotar lo que allí se decía y elaborar un bando. El texto que exponemos a continuación es la transcripción de las anotaciones que hizo una de las alumnas del último ciclo -en calidad de secretaria- con algunas correcciones que se hicieron posteriormente en el aula. No se trata de la totalidad del documento, pero sí de lo más significativo, dado que después hay repeticiones.

A. Expone un tejido hecho en telar por su madre en el campo (Gan Gan, provincia del Chubut). Dijo que las tradiciones quedaron abandonadas y que le gustaría que se renueven. Aclaró que para tejer usaban lana de oveja y pelo de guanaco. (Ver foto 1 ).

Coordinación. Se dijeron algunas palabras sobre los ponchos usados en la época de la Revolución de Mayo, del lugar de los textiles mapuche en los circuitos comerciales a través de los Andes entre los actuales países de Argentina y Chile.

B. [Se presentó vestida de "cartonera" con un sombrero y cajas en la mano] Expone sus actividades como cartonera y su actual trabajo en el Plan Jefas y Jefes de Hogar. Señaló que aún así puede trabajar como cartonera. Otra compañera y vecina la apoya y le dice que es trabajar dignamente. (Ver foto 2).

C. Propone tratar el problema del agua en las calles bajas, de las cloacas y el del barro en las calles con la consiguiente plaga de mosquitos. También habló de la salud. Propone que para cuidar la salud habría que agrandar el desagüe y bajar las calles, y que se haga otro canal. (Ver foto 3).

D. Opina que ocurre lo mismo en el sector que lo rodea, Blandengues, Pacífico, Chaco, Roca, Río Atuel y Rondeau.

E. Pone en consideración el problema de la basura, dice que hay poca recolección y por lo tanto proliferan las ratas. Propone que pongan un contenedor para la basura y que la retiren adecuadamente. También se habla de camiones que tiran basura en la calle.

D. Opina sobre la salud de los chicos en este medio y recordó junto con C. los problemas de las Iluvias y las inundaciones, pero por sobre todo la falta de respuesta de las autoridades cuando se reiteran estas tragedias. Señalaron que se sienten marginados, que llamaron a los bomberos durante una inundación y no llegaron, que tuvieron que ayudarse entre los vecinos.

$F$. Dice que no hay turnos suficiente en la sala médica.

G. Dijo que a ella siempre la atienden en la sala y no le cobran nada. 
H. Quiso hablar de los jubilados y de la poca atención que reciben en la obra social. Dice que no reciben ayuda del gobierno y pide un aumento para los jubilados.

I. Dice que la basura tapa los canales, pero opina que los vecinos también tienen la culpa.

D. Nos informa sobre las características del trabajo de los cartoneros, y el problema de la selección, ya que trabajan de noche y el cansancio impide que puedan desechar la basura rápidamente.

C. Aclaró que ella cartonea con un carro con caballo, cuando Ilega tiene que darle agua y comida al caballo, comer ella misma y descansar para poder salir al otro día a vender el cartón. Señala que le preocupa el tema del canal porque es un problema para los chicos y para los animales que suelen caerse adentro; pero las autoridades no se hacen cargo de estos problemas y los vecinos lo tapan de basura pero no alcanza.

J. Comentó que le preocupa que ahora los chicos no estén interesados por la historia, que ella le pregunta por las fechas patrias pero que no saben nada.

[El grupo opina que deben hacer valer sus derechos como personas, por lo tanto deben encontrar una solución a sus problemas y creen que hasta el momento no han sido escuchados Con estas ideas se elaboraron las conclusiones a las que arribó el cabildo. (Ver foto 4).

Ya dijimos que el texto fue leído y corregido con todos los participantes, a los efectos de verificar que reprodujera lo expuesto en este acto. Gran parte de este encuentro fue filmado, el registro de video fue mostrado en el aula, además se dejó una copia en la escuela.

Con la lectura de este material, surgieron una serie de reflexiones acerca de la importancia del voto para elegir a los funcionarios pero resaltaron las limitaciones que tiene este sistema. Entre las limitaciones más notables del sistema democrático en la ciudad apuntaban que no se puede elegir directamente a los delegados municipales; es así como los ciudadanos eligen al intendente y éste a su vez a los delegados.

\section{c- La elaboración de un cuadernillo}

A pesar de la rápida reflexión que pudo exteriorizar este grupo acerca de sus problemas actuales, fue muy difícil trabajar para construir la historia de la barriada. 
La elaboración en conjunto de un cuadernillo que dé cuenta de la historia y la cultura de los alumnos que asisten a los talleres es siempre una consigna. En este caso, no había intención en el alumnado de trabajar con el pasado, si bien algunas de las alumnas se entusiasmaron con algunos temas, siempre fue difícil la motivación.

Pensamos que el compromiso que asumieron como participantes del taller en la realización del "cabildo" se debió a que se planteaban temas de absoluta actualidad, pero la mirada al pasado desde este presente generaba resistencias. Analizamos estos comportamientos desde nuestra experiencia en otros talleres en los cuales había gran necesidad de abordar el pasado desde la historia social. Resultó notable que las alumnas no tenían interés en trabajar acerca de la historia del barrio o de su propia historia, en especial mantuvieron una negativa muy fuerte para reconocerse como de origen chileno, indígena ${ }^{5} \mathrm{u}$ otros, sin embargo hicieron encendidos alegatos acerca de la legitimidad de trabajar como "cartoneras". En estos talleres, el tema del cartoneo surgió no sólo como una ocupación sino como una cuestión identitaria que las singulariza.

Otra cuestión identitaria que siempre surge en las escuelas es la adscripción religiosa, en general predomina la evangélica pentecostal, tal vez por la institución en la que estábamos (católica); no hubo un reconocimiento de sus opciones religiosas, a las que consideraban un hecho privado que no deseaban hacer público.

Como en todos los casos, el título del cuadernillo se debate, esta vez fue elegido: "Aquellas voces olvidadas de ayer y de hoy". No sólo el título es una afirmación de la existencia de sus voces que tienen una sonoridad que no es escuchada, también lo fueron las palabras introductorias que escribió una de las alumnas:

Las palabras de Claudia

En un barrio transcurre la situación. Se dice que le llaman Bajo Rondeau (a su lado está Villa Caracol, Villa Nocito y San Blas) y que está olvidado.

Es un barrio humilde, tranquilo, con anécdotas y muy querido. La gente que lo habita viene luchando por él hace varios años.

5- En otros talleres hemos trabajado estos temas, festejado el día de la independencia de Chile, recopilado canciones populares, juegos, creencias, vocabularios específicos y profundizamos en la utilización de los recursos alimenticios a través de una historia de las comidas. 
Sueñan que algún día sus hijos lo amen como ellos, que luchen por él como lo han venido haciendo todo el tiempo.

Ellos luchan por ser escuchados, para que se sepa que en él viven personas que quieren vivir dignamente y que pelean por conservar su cultura y su hogar.

Queremos trabajar para tener salud, trabajo, educación, un mundo mejor y queremos que nos escuchen los que gobiernan. En "Aquellas voces olvidadas de ayer y de hoy" aparecen transcriptos los relatos que se registraron en un grabador sobre los siguientes temas:

- "Algo sobre la historia de los barrios Villa Caracol, Bajo Rondeau y San Blas". Los testimonios dan cuenta de cómo fue creciendo el barrio y en especial del lugar que tuvo la recolección de cartón, vidrio y metal. Una de las alumnas se reconoció como primera pobladora de Villa Caracol, hoy denominada "Vista al Mar". ${ }^{6}$

- "Escuela y trabajo". Contaron que no pudieron ir a la escuela porque vivían en el campo y las distancias eran muy grandes, otras fueron pero no pudieron concluir con la educación primaria. También hicimos una breve historia de los trabajos que realizaban las mujeres en el campo y las ocupaciones actuales. A lo largo de los talleres habíamos tratado el tema del tejido en telar, la esquila de chivas y ovejas, el trabajo en los hornos de ladrillos y en la huerta en el ámbito rural. En la ciudad han trabajado eventualmente en el servicio doméstico, en parques y plazas cuando se implementó el Plan Trabajar, en comedores de iglesias de distintos credos como voluntarias o como contraprestación a la recepción de algún plan social y siempre aparece el tema del cartoneo.

- "Comer y curarse". En este caso realizamos un breve recetario con las comidas más representativas de los migrantes chilenos y patagónicos que son muy importantes en todo este sector de la ciudad, así como su articulación con los alimentos que proveen los planes sociales. También se dialogó sobre cuestiones vinculadas a las concepciones de salud/enfermedad y sus demandas al sistema de salud.

6- El nuevo nombre del barrio intenta nombrar de otra manera a la pobreza pero no lo logra, en realidad nos recuerda que están viviendo sobre un terreno salitroso al cual se acerca el agua de mar cuando sube la marea. La cercanía del mar lejos de ser una ventaja agrava realmente las condiciones de quienes viven allí. 
- "Historias para sentir un poquito de miedo". Dedicamos una clase a contar historias increíbles, la mayoría de ellas contextuadas en un entorno campesino que da cuenta de su pasado rural.

- "Las fiestas". Se recordaron las festividades de San Juan en Chile y en la Patagonia argentina. Las fiestas de la Cruz de Mayo y los velorios ${ }^{7}$ en Chile. Por otra parte, aunque no ocupó un lugar en el cuadernillo, siempre salía el tema de la "cumbia villera", ya que eventualmente asistía un adolescente que les recordaba a sus compañeras que él no coincidía con sus gustos musicales. Las alumnas más jóvenes dijeron en más de una oportunidad: "somos villeras y acá la música es la cumbia" o "aguante la cumbia".

\section{Historia en la escuela y participación}

En la rama de adultos la enseñanza de la historia no se considera prioritaria. Por su parte los alumnos de las escuelas sienten a la historia que se enseña como ajena. Algunos maestros suelen acercar los hechos históricos a vivencias y situaciones que permitan que los alumnos se ubiquen en esta historia y en este espacio, pero no hay una propuesta oficial para esta enseñanza. La historia escolar está marcada por el calendario de festejos y conmemoraciones que señala el Ministerio de Educación.

Por otra parte, los maestros no cuentan con material didáctico apropiado para promover la reflexión sobre el ejercicio de los derechos políticos y sociales. Se suma a esta situación que generalmente los anexos escolares funcionan en edificios prestados por distintas instituciones; ambas institucionalidades (la escuela, iglesia, sociedad de fomento) coexisten generándose muchas veces contradicciones entre ambas, ya que los mandatos fundacionales y los intereses del presente suelen contraponerse. En nuestro estudio de caso, la escuela funciona en dos aulas prestadas por la congregación salesiana, el establecimiento se llama "San Dionisio" y allí funcionan además un comedor y un centro de Cáritas. Sin dudas la estética escolar es más religiosa que laica, la dirección y los maestros hacen el esfuerzo diario de hacer "más laica" a la escuela, pero sería impensable una biblioteca, ya que si la hubiera sería básicamente religiosa. Además, en ese entorno una biblioteca sería vis-

7- Incluimos los velorios junto con las fiestas debido a que son recordados ambos por su carácter festivo. 
ta como un lujo, los espacios disponibles son ocupados para actividades de primera necesidad; por ejemplo, este año (2004) han conseguido incorporar un consultorio de pediatría municipal.

Desconcierta la orfandad en la que se encuentran los maestros para enseñar historia a los adultos, los cuales además tienen opiniones formadas acerca del pasado y tienen sus ideas políticas. Aunque si prestamos atención a las palabras de Ezequiel Adamovsky, esta situación no es muy diferente en otros espacios, en los cuáles se necesita de una producción historiográfica adecuada que pueda poner a disposición de los interesados relatos significativos del pasado y no análisis puntuales en los cuales cuesta entender la trama social:

"Si me dispuse, en este trabajo, a rescatar la dimensión de la Historia como actividad vital, fue porque considero que el desempeño de esta función, por parte de la historiografía académica actual se encuentra en peligro. Las producciones universitarias "científicas", juegan un papel en la significación social del pasado. Entendemos que no ha sido una preocupación mayoritaria de los historiadores profesionales las reflexiones sobre la enseñanza de la historia; la producción de un relato del pasado significativo recae en manos, en el mejor de los casos, de historiografías no reconocidas por el campo académico y no cumplen con sus criterios (como el caso de Félix Luna) o de narraciones periodísticas, literarias, cinematográficas, etc. Aún así, la presencia del pasado en la sociedad, no importa su origen, es relativamente escasa, si comparamos con otras épocas" (Adamovsky 2002: 17).

\section{El contexto socio-político en que desarrollamos las experiencias}

En el análisis del presente de la "Educación de Adultos", consideramos que se hace necesario considerar las vinculaciones entre política social y política educativa. Asimismo, es preciso considerar las rupturas en la concepción del "sujeto" en estas políticas. Nos interesa estudiar la cuestión de las transformaciones de las identidades sociales, problemática enmarcada en las nuevas relaciones que se establecen entre estructura y acción (Svampa 2000: 2), el análisis de ambas políticas (en tanto contextos de conformación de la subjetividad), nos permitirá iluminar la cuestión de la transformación de las identidades sociales. 


\section{a- La "educación como trabajo"}

Resulta un fenómeno "novedoso", el hecho de que asistir a la escuela o espacios de formación como Centros de Formación Profesional, Centros de la Mujer o Centros de Alfabetización Municipales, sea remunerado económicamente a través de "planes sociales", ésto es de Políticas Sociales ${ }^{8}$. Las y los trabajadores desocupados (auto-organizados en movimientos políticos y sociales), fueron fundamentales en la gestión de más planes, y los mismos movimientos fueron consolidándose progresivamente ${ }^{9}$. Los debates sobre "educación y trabajo" en trabajadores desocupados, su posible "remuneración" por asistir a espacios públicos de formación (considerando esto como una retribución que bajaría los índices de la pobreza, tal como lo señala el B.M), ya estaban instalados desde una perspectiva neoliberal en el Gobierno Nacional en el año $2000^{10}$.

\section{b- Descentralización educacional}

\section{A partir de nuestro trabajo en educación de adultos contamos con}

8- El Programa "Jefas y Jefes de Hogar" fue implementado por el gobierno Nacional en enero del 2002 con el apoyo de un préstamo del Grupo Banco Mundial (en adelante B.M) que cubrió el 50\% de su financiamiento, "como respuesta a la crisis económica que afectó al país en diciembre del 2001", tal como lo señalan los documentos del B.M en la página oficial en internet (Galasso y Ravallion 2003). Pensado como continuidad del plan "Trabajar" (también financiado por el B.M) el "Plan Jefes y Jefas" preveía el otorgamiento de dinero en efectivo por 150 pesos a cada "cabeza de familia" desocupado /a con hijos o ancianos a cargo. Se preveía como contraprestación del mismo 20 horas de trabajo (diez menos que el Trabajar), y se accedía al plan inscribiéndose -en forma individual- en las municipalidades u ONGs locales (que eran quiénes estaban encargadas de organizar los trabajos de contraprestación). Según datos del propio B.M., la mitad de los inscriptos en 2002 eran mujeres, y en función de este dato cuestionan la efectividad de los planes poniendo en duda la veracidad de la generalizada jefatura de hogar femenina (Galasso y Ravallion 2003:2).

9- Tanto movimientos piqueteros preexistentes al estallido del 19 y 20 de diciembre de 2001, como otros que se organizaron luego, en Bahía Blanca, la CCC, El MTL y el POLO OBRERO.

10- En Agosto del 2000 asistimos en el I Congreso Internacional de Educación en la Facultad de Filosofía y Letras de la U.B.A. a un Panel sobre "La educación en la cuestión social" donde participaron Tomaz Tadeu da Silva, Emilio Tenti Fanfani y Aldo Issuani, éste último funcionario del Ministerio de Educación del Gobierno Nacional. Generó gran polémica la intervención del citado funcionario al considerar que "el estudio es un trabajo y viceversa", pensando en reducir en 1/3 la tasa de desempleo si se afectara con un programa a los sectores más vulnerables, mujeres con chicos a cargo y jóvenes sin escolaridad secundaria completa desocupados, y que por ello era justo que se remunere, ya estaban en vigencia los planes Trabajar. Se cuestionaba la sobrevaloración de la medida, que sólo era un paliativo y no la solución más digna a la situación de indigencia en que vive la mitad de la población del país. 
indicios que nos permiten señalar que esta "rama", "régimen" (en la Ley Federal de Educación) ha sido "pionera" en los proyectos neoconservadores en educación. Señalada como "residual" en la Ley Federal de Educación (Monteverde 1996: 88), entendemos que las políticas implementadas en la misma rama han sido un antecedente de la misma Ley y sus consideraciones acerca del Polimodal, con la creación de los CENS ${ }^{11}$ en el año 1985. En el período de implementación de la Ley Federal de Educación en la provincia de Buenos Aires, si bien la rama de adultos es considerada residual en la Ley Federal (incluida dentro de Regímenes Especiales y que aún no desarrolló lineamientos curriculares), avanzaba en políticas de articulación productiva entre la Dirección General de Cultura y Educación y el Municipio, puntualmente a través de "Formación Profesional".

El capital bancario y monopolista y la burguesía internacional (representados en el Banco Mundial) venían diseñando lineamientos sobre educación ya a fines de la década del 80 , cuando planteaban la no rentablidad de la educación y la necesidad de "recuperar costos", la descentralización y apoyo a las escuelas privadas y comunitarias. Asimismo (y en esta lógica) la Ley Federal de Educación del año 1993 representa una avanzada en estas concepciones, así como los documentos del Banco Mundial de 1996 y los posteriores a nivel nacional y provincial como lo fue el resistido y finalmente, no implementado, Pacto Federal Educativo II en el año 2000, o el decreto de creación de las Unidades de Planificación del Desarrollo Educativo Distrital, creadas en el año 2001 (conformadas en el presente). A fines del 2001, la fundación FIEL "sugirió" medidas para avanzar en la autogestión de las escuelas. El sujeto de la educación en estas propuestas es un "cliente", así como hay una concepción "individual" del trabajador de la educación y del trabajo docente. La sociedad es vista desde una perspectiva funcionalista como conformada por diferentes "agentes" y sostiene la concepción de un Estado "débil" y al mismo tiempo "controlador" de los movimientos socia-

11- Centro de Educación de Nivel Secundario, cuya creación es a partir de 1985 una facultad del Ministerio de Educación, mediante la suscripción de convenios entre la Dirección de Educación de Adultos (DINEA) e Instituciones de la sociedad (Decreto 101/85). Con la transferencia de 1991, la ahora, Dirección de Educación de Adultos (D.E.A.), según decreto No 1392/91 se facultada al Ministerio de Educación para "suscribir convenios, con autoridades nacionales, provinciales, municipales, instituciones públicas o privadas de bien común, ambas partes coordinando las formas de acción que permitan su implementación"(la de los CENS). Claro antecedente de lo que la Ley Federal de Educación sentaría para el polimodal y su dictado en "instituciones específicas". 
les, con una presencia fuerte en lo que hace al control de los recursos y, en definitiva, de los movimientos y agrupaciones de la sociedad (control de los planes, desentendimiento de responsabilidades respecto de la educación). Ambas cuestiones claves en las tendencias necoconservadoras en educación: descentralización (derivación de responsabilidades en los municipios) y articulación entre mercado y educación tienen en la rama de adultos del sistema educativo provincial lamentables antecedentes.

\section{adultos \\ c- Los movimientos sociales, teorías pedagógicas y educación de}

El contexto en que emergen las políticas y las teorías pedagógicas que sostuvieron el surgimiento de la escuela en nuestro país, se caracterizó por la presencia -en el campo social y político-, de sujetos sociales críticos del sistema capitalista. Desde la pedagogía oficial se hacía preciso negar a estos sujetos. La diversidad de experiencias y sentidos que desde comienzos del siglo XIX sostenían a la Educación de Adultos desde movimientos anarquistas, socialistas, sociedades populares de educación, etc. derivó avanzado el siglo (puntualmente en la década del '20) en la consolidación de una propuesta totalizadora, que se llevó a cabo bajo la hegemonización de aquella multiplicidad que la sociedad civil había generado (Rodríguez 1996:81). En las discusiones y debates previos a la ley 1420, a fines de siglo XIX, se fue consolidando el discurso que le da fundamento al proyecto oligárquico: una concepción racista que se habría ido renovando desde la conquista, trazando una continuidad del debate acerca de la educabilidad del indio a los inmigrantes europeos (Puiggrós 1992). Tal como lo plantean Neufeld y Thisted, "hoy los extranjeros son otros: bolivianos, peruanos, paraguayos y chilenos, coreanos, chinos, rusos y ucranianos. Una estratificación naturalizada y compartida" (Neufeld y Thisted 1999:27), una estratificación que guarda continuidades con aquella historia, rupturas y resignificaciones "que tienen que ver con los cambios político-económicos y culturales del siglo XX" (Neufeld y Thisted 1999:28). Estos autores señalan que no corresponde a las condiciones actuales la metáfora del crisol de razas sino más bien se da una exhibición balcanizada de la diversidad acompañada de una naturalización de distintos procesos de exclusión.

En este contexto, planteamos que es preciso insistir, las prácticas pedagógicas preceden al surgimiento de la escuela, por un lado la pedagogía de 
los pueblos originarios, negada, silenciada como los mismos pueblos, por el otro la pedagogía colonial, de raíces monásticas, sea en conventos, sea en los cabildos y por otros, la pedagogía socialista y la pedagogía anarquista que Ferrer Guardia nos narra en La Escuela Moderna, a principios del XX, experiencias por fuera de los muros de las escuelas (Barrancos 1997; Rodríguez; 1996) que confrontó con la pedagogía escolar del Positivismo y la Escuela Nueva, con las cuales en nuestro país Aníbal Ponce sostuvo un importante debate en la década del '30 (Ponce 1950; Tamarit 1992). Se da un espiral de encuentros y desencuentros de los pensamientos de los pueblos acerca de la formación, y conviven pensamientos y prácticas caracterizados por poner la educación al servicio de proyectos sociales contrapuestos, pedagogías al servicio de los poderosos o al servicio del pueblo. Consideramos que las teorías de la reproducción iluminaron un amplio cuerpo de prácticas sociales que permanecían ocultas bajo la lupa de las teorías defensoras de la "escolarización". Categorías como "arbitrario cultural dominante" y "habitus", desarrollados por Bourdieu y Passeron señalaron el problema del poder en las prácticas pedagógicas y las vinculaciones dialécticas entre la subjetividad y las estructuras sociales (Bourdieu y Passeron 1970; Tamarit 1992). La pedagogía crítica ha recuperado la perspectiva gramsciana del poder, sobre todo el concepto de "hegemonía", así como las conceptualizaciones de Foucault acerca del carácter "productivo" del poder (Tadeu da Silva 1995:109).

Por último, articular los planteos heredados de Antonio Gramsci con las reflexiones de pensadores latinoamericanos como Mariátegui y Freire sería de gran valor para la teoría pedagógica latinoamericana. Hegemonía, contrahegemonía y hegemonías alternativas se hallan espiraladas en la historia de la educación latinoamericana. Con Paulo Freire pensamos y sentimos que es preciso seguir imaginando prácticas pedagógicas liberadoras.

\section{Las metodologías participativas en historia}

García y Batallán nos han aportado conceptos desde la antropología para pensar acerca de esta metodología de investigación participativa. Los espacios de co-investigación grupal tienen como intención el promover un proceso de conocimiento reflexivo sobre su cotidianeidad en relación a las problemáticas planteadas. Tanto interesa el conocimiento de los procesos sociales como la resignificación que la misma investigación desencadena en los sujetos partícipes de esa realidad. Esta relación social implicada en el en- 
cuentro de investigación se convierte en forma y contenido (García y Batallán 1994: 168). En estos espacios, el material con que trabajamos, refleja tanto la textualidad, como el contexto y los significados atribuidos por los sujetos a los acontecimientos pasados o presentes. Los contextos en que trabajamos están constituidos por ideologías institucionales, siendo entonces susceptibles de interpretaciones polémicas.

Ha sido importante para la construcción de estos espacios el interés de los participantes por incluirse en este proceso de indagación. Este proceso intenta que los sujetos puedan poner en cuestión la realidad, desarticulando el sentido común, el discurso obvio. La crítica grupal de la visión subjetiva de la realidad permite que los sujetos sean intérpretes "insoslayables" de la realidad en estudio y por otra, esta misma interpretación debe ser considerada y analizada en su heterogeneidad. A nuestro entender este es un imperativo ético y político. El conocimiento será liberador en la medida en que permita “disolver actitudes dogmáticas provenientes de la dificultad para elaborar los conflictos" (García y Batallán 1994:171). Estos autores realizan la analogía entre este proceso grupal y una "gran entrevista abierta" en la que mediante un diálogo simétrico entre el coordinador-investigador y los participantes se construye un cuerpo hipotético para explicar la realidad a partir de una red de sentidos compartidos que es "penetrada" por el análisis reflexivo.

Los procedimientos de contrastación de la información por triangulación, las oposiciones entre el sí mismo y los otros, entre momentos vivenciales y diferenciales de la propia historia y finalmente la participación de los mismos sujetos partícipes de la investigación en las instancias de validación (García y Batallán 1994:172) son parte de esta metodología participativa de trabajo en historia oral en que nos hemos comprometido a trabajar.

En el campo de la investigación educativa, y particularmente en educación de adultos, Teresa Sirvent, ha contribuido a generar experiencias y reflexiones acerca de investigación participativa en distintos contextos en Latinoamérica y, en particular en Buenos Aires (1984; 1994; 1999). Esta autora considera que la investigación participativa es una metodología generada en América Latina en un momento específico y dentro de un contexto de cuestionamiento profundo en el campo de las ciencias sociales, por lo que va diseñándose como respuesta a condiciones objetivas de determinado momento histórico (1994:66). La participación no es un 'juego', nos dice Sirvent, "implica la necesidad de ruptura de representaciones colectivas e ideologías cotidianas y significa un proceso de aprendizaje a través del cual 
se cuestiona y se adopta una conciencia crítica de nuestro sentido común" (Sirvent 1994:74).

Interrogantes que están presentes en nuestro trabajo son aquellos vinculados al ¿cómo se elabora la construcción colectiva?, ¿cómo se reflexiona sobre la práctica del grupo involucrado? y ¿cómo es posible socializar el conocimiento producido? (Sirvent 1994:75). En este proceso de caminar preguntando, hemos retomado los conceptos básicos y variables que Sirvent propuso para el análisis de la participación real y la simbólica: quién participa, cómo, a través de qué mecanismos y en qué ámbitos. En las respuestas a estos interrogantes están presentes los condicionamientos institucionales, las estructuras elitistas de poder, que suponen la concesión. Entendemos que la participación real "constituye un largo y difícil proceso de aprendizaje de conocimientos, actitudes, habilidades y destrezas que modifique los modelos de relación humana internalizados en años de autoritarismo y explotación" (Sirvent 1994: 47).

La investigación participativa es una metodología en construcción; ha supuesto una serie de rupturas, en principio supone una relación dialéctica entre sujeto y objeto de investigación y enseñanza y aprendizaje. Esto implica quebrar la relación unidireccional y jerárquica que ha existido entre el sujeto y el objeto de la investigación o el aprendizaje e incorporar variables que hacen a la subjetividad interpretativa de quienes se sienten involucrados en procesos sociales. Isabel Hernández señalaba que éste es un tema crucial, del que dependerá la posibilidad del educador y del investigador de reconocerse a sí mismo involucrado en esta unidad de opuestos, entre educador y educando, entre sujeto y objeto de la investigación (Hernández 1985: 31).

Esta metodología de investigación y de praxis política suponen la superación de la posición ambigua frente a la relación sujeto-objeto, en el intento de generar, no solamente una mera reflexión teórica, Vera Gianotten y Tonn de Witt proponen analizar el concepto de intelectual orgánico gramsciano, a fin de esclarecernos y asumir nuestro papel y función en la práctica concreta: participar e insertarse en el proceso histórico de organización del pueblo, para que éstos puedan generar sus propios intelectuales orgánicos y a la vez, asimilar al intelectual comprometido (Gianotten y de Witt 1985:136).

\section{5- La metodología de la historia oral en historia de la educación}

La historia oral está siendo empleada como metodología privilegiada 
para la realización de nuestro estudio de la historia social de la educación de campesinos migrantes en la ciudad de Bahía Blanca. Compartimos el planteo de que es preciso recuperar la oralidad como vía de simbolización, como organización semiológica colectiva por los trabajos en historia y, que es preciso considerar el lugar único, incomparable que tiene el habla, la oralidad en la experiencia humana (Barrancos 1997:158).

La noción de pluralidad cultural es el principal componente de los fundamentos de los procesos educativos latinoamericanos, esta mirada nos permite pensar en otro sujeto pedagógico diferente al de la tradición liberal en educación (Puiggrós 1997:114). La relación pedagógica fundante de dominación, representada en la escena del Requerimiento, con su carácter autoritario e impositivo ha dejado una marca, y somos descendientes de ésta; ha fundado nuestra identidad presente, como herida que no cesa de volver (Puiggrós 1997:114).

La historia de la educación fue construida casi exclusivamente a partir de la historia de la escuela; estudiar la de los pueblos desde un enfoque latinoamericanista requiere indagar los modos de producción y transmisión de saberes, que exceden la lecto-escritura. La relación entre la aparición de la escuela, la adquisición de la lecto-escritura y la promoción de la lectura no es una relación lineal para los historiadores de la lectura, y sigue siendo motivo de indagación (Coock Gumperz 1988). Es preciso reconocer las relaciones de lucha por la hegemonía al interior de lo pedagógico, lo que implica no descalificar la institución escolar por lo que hemos trabajado en torno de las historias de los mismos sujetos en las escuelas (Puiggrós 1997: 110).

Nos interesa revalorizar la noción de saber popular, considerando que las nociones de "saber popular" o "sentido común" no son unidades homogéneas pertenecientes a las clases subalternas, opuestos al saber hegemónico de las clases dominantes (García y Batallán 1994: 168). Entendemos que el sentido común se va construyendo de modo contradictorio, aunque guarda coherencia y sentido desde el punto de vista de su elaboración; es una respuesta colectiva y tiende a reorientarse por la acción que ejercen los aparatos de hegemonía (Paoli 1984: 25, Tamarit 1997:130). Núñez señalaba la necesidad de concretar, entre las líneas de investigación en Historia de la Educación, a la educación del pueblo (en Puiggros 1997: 115), compartimos tal planteo entendiendo que es preciso siempre definir en cada momento histórico al sujeto del pueblo y las formas posibles de su constitución como fuerza social efectiva (Tamarit 1992: 58), en la perspectiva del conflicto po- 
demos hablar de las clases dominadas (o "no dominantes").

En nuestra experiencia de historia oral en talleres, hemos intentado recuperar los conflictos y las luchas entabladas entre:

- Propietarios y peones: por el espacio, dónde podían y no podían jugar los niños, la propiedad del patrón como intransitable, sólo para prestar la fuerza de trabajo. El trabajo en las minas en la juventud y la represión de la organización sindical. Los mestizos e indígenas como fuerza de trabajo en las minas y en el campo, así como de empleadas domésticas en casas de propietarios (muchas veces extranjeros). Escuelas diferenciadas para los hijos de patrones y de peones.

- Estado-iglesia-escuela, campesinos y pueblo originario: la escuela no estaba en la infancia a disposición de los mismos tanto "espacialmente" como en función de los contenidos transmitidos. El conocimiento popular y la institución escolar han estado -y consideramos que en alguna medida siguen estando- en permanente conflicto. Exclusión abierta o inclusión excluyente han sido y son las estrategias. Como parte de la actividad de los talleres, además de los relatos referidos acerca del saber popular hemos propiciado la producción de dibujos acerca de los lugares de residencia, juegos y alimentos, como también la resignificación de obras de pintores como Diego Rivera y Antonio Berni.

- Hombres y mujeres: los aprendizajes han sido poco diferenciados. Hay particularidades como el aprendizaje del hilado y tejido que ha sido casi exclusivo para las mujeres. Varones y mujeres han compartido las tareas rurales. Las mujeres han sido mayormente excluidas de la posibilidad de apropiación de la lecto-escritura y los hombres muchas veces lo hicieron en el servicio militar.

\section{6- Los planteos sobre la enseñanza y el aprendizaje de la historia}

Tadeu Da Silva ha señalado la posibilidad que la pedagogía crítica tiene de "descolonizar el currículum", en tanto la educación, el conocimiento, el currículum y la noción de "contenidos" misma, no son cosas sino que "corporifican relaciones" (1997:30), resaltando el contenido social de los mismos. De este modo considera la noción de "relaciones sociales" como dispositivo pedagógico. Insertar en el marco de conexiones sociales más amplias a la educación y el currículum, considerar su carácter construido y relacional, 
considerando los procesos de fetichización y reificación y el carácter particular e histórico del conocimiento y el currículum. Estos planteos nos han servido de sostén en nuestro trabajo de historizar las prácticas educativas en el contexto escolar intentando favorecer la apropiación de los sujetos de su propia historia. El planteo de descolonizar el currículum nos permitió promover en el marco de la experiencia de participación en talleres de historia oral en la escuela, la visualización de los testimonios a partir de las relaciones de clase, las consideraciones acerca de las relaciones de dominación entre naciones, de subordinación étnica y las relaciones asimétricas entre los géneros. Estas categorías nos han servido de soportes teóricos para analizar los propios relatos dando cuenta del carácter no natural de la desigualdad, sino un producto social en el marco de relaciones sociales de poder.

Funes plantea en la indagación acerca de las concepciones sobre la enseñanza de la historia e interroga: ¿Qué historia enseñar?, pregunta que Ileva a hablar sobre cuestiones epistemológicas en el conocimiento histórico implica que la reflexión sobre la condición humana (Funes 2003:166). Estas cuestiones, vinculadas al ¿para qué enseñarla?, depende para su respuesta de la concepción epistemológica, de los contextos socio-históricos y de los proyectos político-educativos (2003:167).

Recientemente Pagès retomaba investigaciones de Jensen, quién señala que en la formación docente en historia se ha tomado conciencia de que los niños "aprenden mucha historia fuera de los muros de la escuela" (Pagès 2003:19), lo cuál contribuyó a reflexionar acerca de las finalidades de su enseñanza siendo el objetivo desarrollar en él la conciencia histórica, entendiendo a la misma como la "interconexión de interpretaciones del pasado no sólo en la comprensión del presente sino igualmente con la concepción de aspiraciones referidas al futuro" (Pagès 2003:20). Funes señala que la conciencia histórica nace siempre de una inquietud sobre el presente y sobre un proyecto para el futuro, y que los propósitos que guían los proyectos son los que orientan las preguntas sobre el pasado; y señala que esta construcción simultánea del futuro proyectado y del pasado reconstruido no es una tarea individual sino social (Funes 2003:153).

Hemos indagado en las propuestas oficiales para la enseñanza de la historia en "adultos" (Hernández y Visotsky; 2003) y nos preguntábamos "¿Qué es la historia en los distintos discursos que se cruzan?" . Hay contenida en el discurso de los funcionarios provinciales de la rama y funcionarios municipales, una concepción de la historia para la que el sujeto de la misma 
son las innovaciones tecnológicas, en este sentido el sujeto es la economía, el modelo económico. En esta línea aparecen desdibujados los hombres y mujeres, estudiantes de las escuelas de adultos, en tanto sujetos de aprendizaje y sujetos de la historia, quienes quedan reducidos a la categoría de objetos, son: "recursos humanos". Nombrados también "esta gente" o "los chicos, una "mano" para la obra ... El militante, el padre, la madre, la militante, las y los trabajadores quedan invisibilizados, infantilizados. Se omite preguntar cuál es su historia, cómo vive, por qué, en qué espacios participan, cuáles son sus problemas y sus proyectos.

Hasta el momento no han sido elaborados lineamientos curriculares para la rama. Se ha sugerido a los docentes la adecuación de los CBC para la EGB. En el año 1997 el Ministerio de Educación de la Nación elaboró materiales didácticos para la "terminalidad"; ésto es educación a distancia. Fueron elaborados y distribuidos por el Plan Social (Plan Social Educativo; 1997). En estas cartillas podemos ver que la organización de los contenidos tiene en cuenta revisiones de las conceptualizaciones en las ciencias sociales realizadas por la didáctica de las ciencias sociales, como el abordar la historia a partir del sujeto, del presente, y el abordaje a partir de conceptos tales como el carácter multicausal de la experiencia histórica (Plan Social Educativo; 1997(a)(b)). Frente a esto, el confuso empleo del concepto de "proceso histórico" en los CBC para Ciencias Sociales de EGB cumple la función teórica de describir, encadenar hechos construyendo cronologías ordenadas por un orden "cuasi natural", asegura una separación entre el sujeto cognoscente y el objeto a conocer y cumple la función social de legitimar las permanencias, el orden social vigente. En el presente año han sido desarrollados documentos oficiales sobre alfabetización de adultos, con un espacio dedicado a la enseñanza de las Ciencias Sociales (DGCyE; 2004), el mismo si bien realiza una apertura a otras fuentes y metodologías desestimadas por la historiografía positivista, la periodización es política; los objetivos propuestos para el abordaje de la disciplina propician la "significatividad" de los contenidos a trabajar, pero no considera el enfoque a partir de la percepción del futuro, esto implicaría concebir a los estudiantes en tanto sujetos sociales; consideramos que a pesar de revisar experiencias en distintos contextos en Latinoamérica, incluso experiencias en contextos revolucionarios, esta propuesta de la provincia no se enmarca en una perspectiva crítica de la educación y de la realidad social, ya que no basta con la ampliación de fuentes y metodologías si no se produce un cambio en el para qué, a favor de quién y de qué, y en 
contra de quién y de qué están pensadas dichas propuestas.

\section{7- Palabras finales}

Hemos reflexionado acerca de las relaciones entre enseñanza y aprendizaje de la historia, a partir de un trabajo de campo realizado durante el año 2003 en una escuela provincial de adultos en la ciudad de Bahía Blanca.

Le dimos mucha importancia a las relaciones entre cultura e historia ya que la cultura de los sectores populares tiende a hacerse invisible y por lo tanto a ser negada por la escuela. Junto con la negación de su cultura los alumnos de las escuelas primarias de la rama de adultos ven negada su historia y sienten que se les enseña una historia que les es ajena.

También incluimos la variable políticas públicas para abordar el tema propuesto. Intentamos analizar cómo influyen las propuestas de descentralización educativa y los planes sociales en esta rama, ya que los alumnos/as adultos/as mayores (la mayoría son mujeres) asisten a la escuela como contraprestación de la recepción del Plan Jefas y Jefes de Hogar.

Por último, quisimos plantear algunas relaciones entre pobreza, políticas públicas y educación basándonos en situaciones empíricas concretas. Consideramos que los condicionamientos macroestructurales de la globalización se cruzan con las condiciones de existencia concretas, en este caso nosotros las estamos observando desde un sector de la ciudad donde la principal fuente de ingreso es el cartoneo, la recolección y selección de elementos que han sido tirados porque se los considera desechos y no bienes aprovechables. Estos condicionamientos generan nuevas adscripciones identitarias a las que pudimos abordar a partir del trabajo en "Talleres de Historia".

\section{Resumen}

La alfabetización de adultos es un tema que concierne a la educación, a las políticas públicas y no es ajena a los planteos acerca de la enseñanza y los aprendizajes de la historia.

El tema de la alfabetización ha sido una preocupación de historiadores como Gramsci y los miembros de la Escuela de Birmingham y de pedagogos como Paulo Freire. Todos ellos partían de la necesidad de conocer la cultura de los alfabetizandos para propiciar que el proceso de alfabetización -aunque no siempre era formal- permita a los sectores no hegemónicos decir sus propias palabras, no repetir palabras. 
Nosotros reflexionamos acerca de las relaciones entre la cultura de los sectores populares urbanos, la historia, la enseñanza y el aprendizaje de la historia a partir de un trabajo de campo realizado durante el año 2003 en una escuela provincial de adultos en la ciudad de Bahía Blanca. Compararemos este material con el recopilado en otros similares realizado también en Centros de Alfabetización Municipal. Nos interesa analizar como influyen las propuestas de descentralización educativa y las políticas públicas en esta rama, ya que los alumnos/as adultos/as mayores (la mayoría son mujeres) asisten a la escuela como contraprestación de la recepción de planes sociales.

\section{Palabras claves}

Alfabetización de adultos - enseñanza de la historia - políticas públicas.

\section{Abstract \\ Literacy of adults is an issue related to education and to public poli- cies and it is no stranger to the teaching and learning of history.}

The topic of literacy has been a worry for historians such as Gramsci and other members of the School of Birmingham and to pedagogues such as Paulo Freire; all of them have had as a starting point the idea that it was necessary to know the culture of the learners in order to make the process of literacy -although not always formal- allow the non-hegemonic people to express themselves in their own words, instead of repeating words. We reflect on the relationship between the culture of populated urban areas, history, the teaching and the learning of history starting from a fieldwork done in 2003 in a provincial adult school in the city of Bahía Blanca. We will compare this material to the information compiled in other similar works done in the Municipal Literacy Centers. We are interested in analyzing the influence of proposals for educational decentralization and the public policies in this area, since adult students (most of which are female) attend school in exchange for receiving social plans.

\section{Key words}

Literacy of adults - teaching history - public politics. 


\section{Bibliografía}

*ADAMOVSKY, Ezequiel (2001), Historia y sentido. Exploraciones en teoría historiográfica. Buenos Aires, El cielo por asalto.

*BARRANCOS, Dora (1997), "Problemas de la 'historia cultural'. Triangulación y multimétodos", en: CUCUZZA, Rubén (comp.), Historia de la Educación en Debate, Buenos Aires, Miño y Dávila, pp. 147-169.

*BARRANCOS, Dora (1997), "Socialistas y la suplementación de la escuela pública: la Asociación de Bibliotecas y Recreos infantiles (1913-1930)", en: MORGADE, Graciela (comp.), Mujeres en la educación, Buenos Aires., Miño y Dávila., pp. 130-150.

*BOURDIEU, Pierre y PASSERON, Jean Claude (1970), La Reproducción, Madrid, Laia.

*COOCK GUMPERZ, Jenny (1988), “Alfabetización y Escolarización: ¿Una ecuación inmutable?”, en: COOCK GUMPERZ, Jenny, La construcción social de la Alfabetización, Madrid, Paidós, pp. 31-59.

*DIRECCION GENERAL DE CULTURA Y EDUCACION DE LA PROVINCIA DE BUENOS AIRES (2004), Alfabetización inicial para jóvenes y adultos, La Plata.

*FUNES, Alicia Graciela (2003), "Significaciones de la historia enseñada", en: Reseñas de enseñanza de la historia, Córdoba, APEHUN, pp. 151-176.

*GALASSO, Emanuela y RAVALLION, Martín ( 2003) "Social Protection in a Crisis: Argentina's Plan Jefes y Jefas", en: Development Research Group - World Bank, wwww.worldbank.org, Washington DC.

*GARCÍA, José y BATALLÁN, Graciela (1994), "Antropología y participación. Contribución al debate metodológico”, en: GARCIA, José, La racionalidad en política y en ciencias sociales, Buenos Aires, CEAL, pp. 162-175.

*GIANOTTEN, Vera y DE WITT, Ton (1985), "Orientación de la investigación participativa hacia la practica: el papel del intelectual orgánico", en: HERNANDEZ, Isabel, Saber popular y educación en América Latina, Buenos Aires, Ediciones Búsqueda - CEAL, pp. 128-138.

*HERNANDEZ, Graciela y VISOTSKY, Jessica (2001). Cultura popular. Cultura indígena. Una experiencia de educación popular en escuelas de adultos, Bahía Blanca, Museo Histórico Municipal.

*HERNANDEZ, Graciela y VISOTSKY, Jessica (2003), “ “La Historia' y 'las historias' en las Escuelas de Adultos”, en: Revista Reseñas de Enseñanza de la Historia, $\mathrm{N}^{\circ} 1$, APEHUN, Córdoba, Universitas, pp. 43-96.

*HERNÁNDEZ Isabel (1985), "La educación popular en la Argentina", en: 
*HERNANDEZ, Isabel, Saber popular y educación en América Latina, Buenos Aires, Ediciones Búsqueda - CEAL, pp. 17-35.

*MONTEVERDE, Ana (1996), "Entrevista a Silvia Brusilovsky. Educación de adultos ¿problemática significativa?”, en: Revista IICE, Año 5, $N^{\circ} 8$, Facultad de Filosofía y Letras, UBA, pp.86-91.

*NEUFELD M. Y THIESTED, J, (1999), “El 'crisol de razas' hecho trizas: ciudadanía, exclusión y sufrimiento”, en NEUFELD, María Rosa y THISTED, Jens (Comp.) De eso no se habla...los usos de la diversidad sociocultural en la escuela, Buenos Aires, Eudeba, pp.23-56.

*PAGÈS, Joam (2003), "Ciudadanía y enseñanza de la historia”, en: Reseñas de enseñanza de la historia, Córdoba, Universitas, APEHUN, pp. 11-44.

*PAOLI, Antonio (1984), La lingüística en Gramsci, Puebla, Premià La Red de Jonás.

*PONCE, Aníbal (1950), Educación y lucha de clases, Buenos Aires, Editorial Iglesias y Matera S:R:L.

*POZO, J. (1994), "El cambio conceptual en el conocimiento físico y social: del desarrollo a la instrucción”, en: A. Rodrigo, Contexto y desarrollo social, Síntesis 4.

*PUIGGROS, Adriana (1992), Sujetos, Disciplina y Currículum en los orígenes del sistema educativo argentino (1885-1916), Buenos Aires, Editorial Galerna.

*PUIGGROS, Adriana (1997), "Presencias y ausencias en la historiografía pedagógica latinoamericana”, en: CUCUZZA, Rubén (comp.), Historia de la Educación en Debate, Buenos Aires, Miño y Dávila, pp. 91-123.

*RODRIGUEZ, Lidia (1996), "Educación de Adultos (E.A) y Actualidad. Algunos elementos para la reflexión”, en: Revista del IICE, Año 5, $N^{\circ} 8$, Facultad de Filosofía y Letras, UBA, pp. 80-85.

*SIRVENT, María Teresa (1984), "Estilos participativos: sueños o realidades", en: Revista Argentina de Educación $N^{\circ}$, Asociación de Graduados en Ciencias de la Educación, pp. 46-59.

*SIRVENT, María Teresa (1994), Educación de Adultos: investigación y participación, Buenos Aires, Libros del Quirquincho.

*SIRVENT, María Teresa (1999), Cultura popular y participación social. Una investigación en el barrio de Mataderos (Buenos Aires), Madrid, Miño y Dávila. *SVAMPA, Maristella (2000), Desde abajo. La transformación de las identidades sociales, Buenos Aires, Biblos, Universidad Nacional General Sarmiento. *TADEU DA SILVA, Tomaz (1995), Escuela, conocimiento y currículum, Buenos Aires, Miño y Dávila. 
*TADEU DA SILVA, Tomaz (1997), "Descolonizar el currículum. Estrategias para una pedagogía crítica", en: Crítica Educativa, $N^{\circ} 2$, Buenos Aires, Miño y Dávila, pp. 17-33.

*TAMARIT, José, (1992), Poder y Educación Popular, Buenos Aires, El Quirquincho.

*VISOTSKY, Jessica, (2003) “Analfabetismo-alfabetización en Bahía Blanca. Conocer: identidad y cambio", en: GADOTTI, Moacir, GÓMEZ, Margarita y FREIRE, Lutgardes, Lecciones de Paulo Freire cruzando fronteras: experiencias que se completan, Buenos Aires, CLACSO-IPF, pp. 161-209.
Abreviaturas
CBC. Contenidos Básicos Comunes
EGB. Educación General Básica
BM. Banco Mundial

\section{Recibido: 28/07/04 \\ Aceptado: 10/11/04}




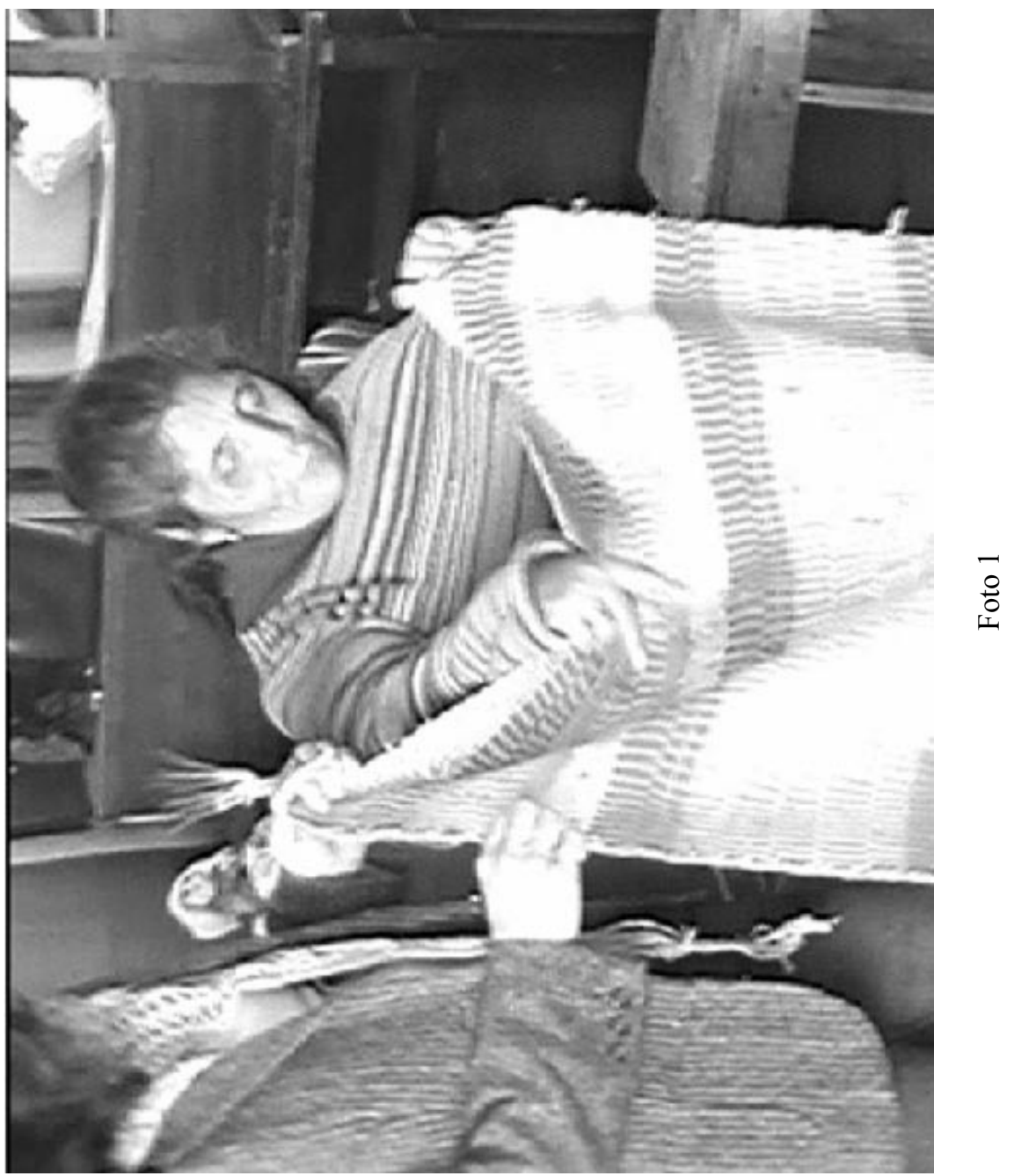





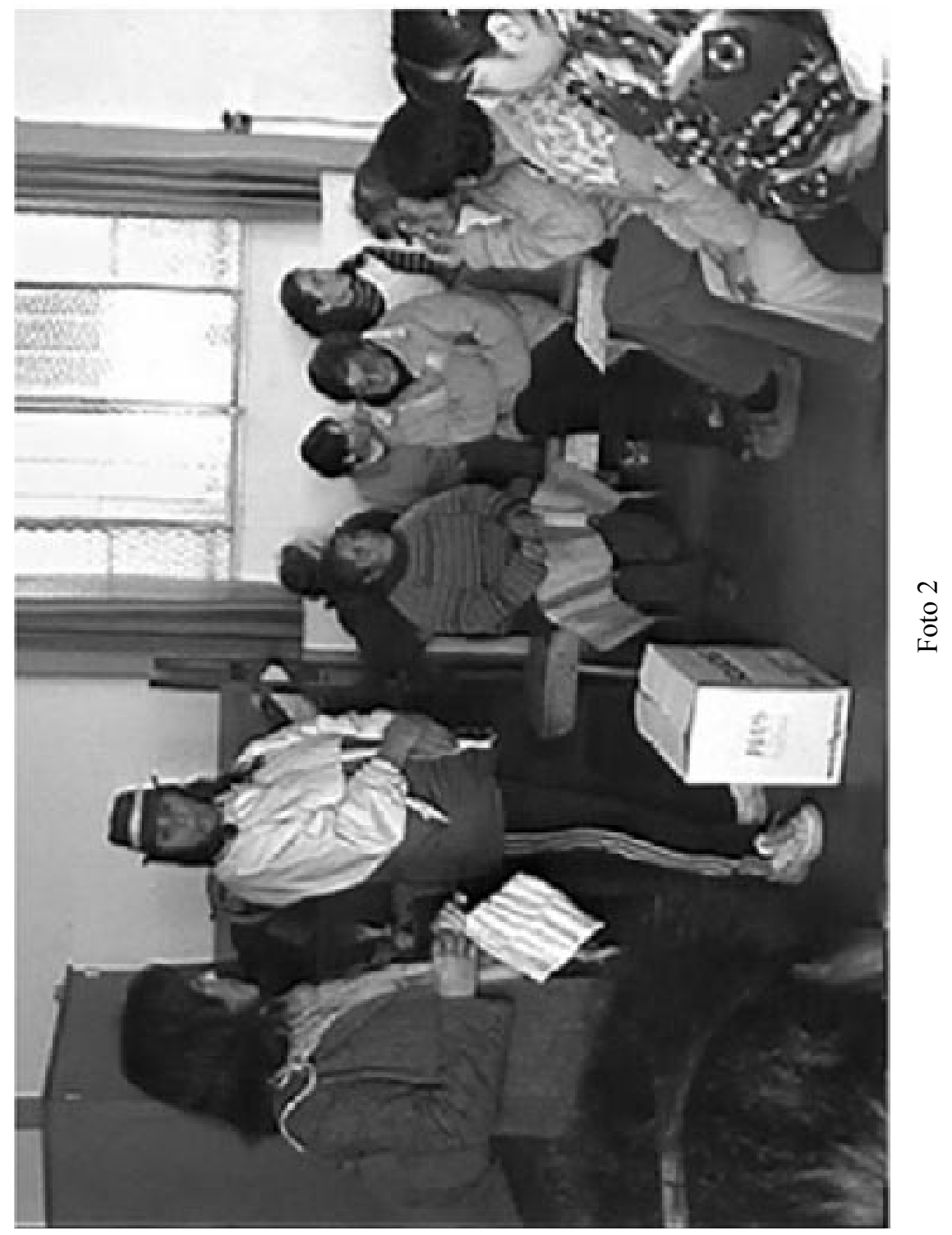





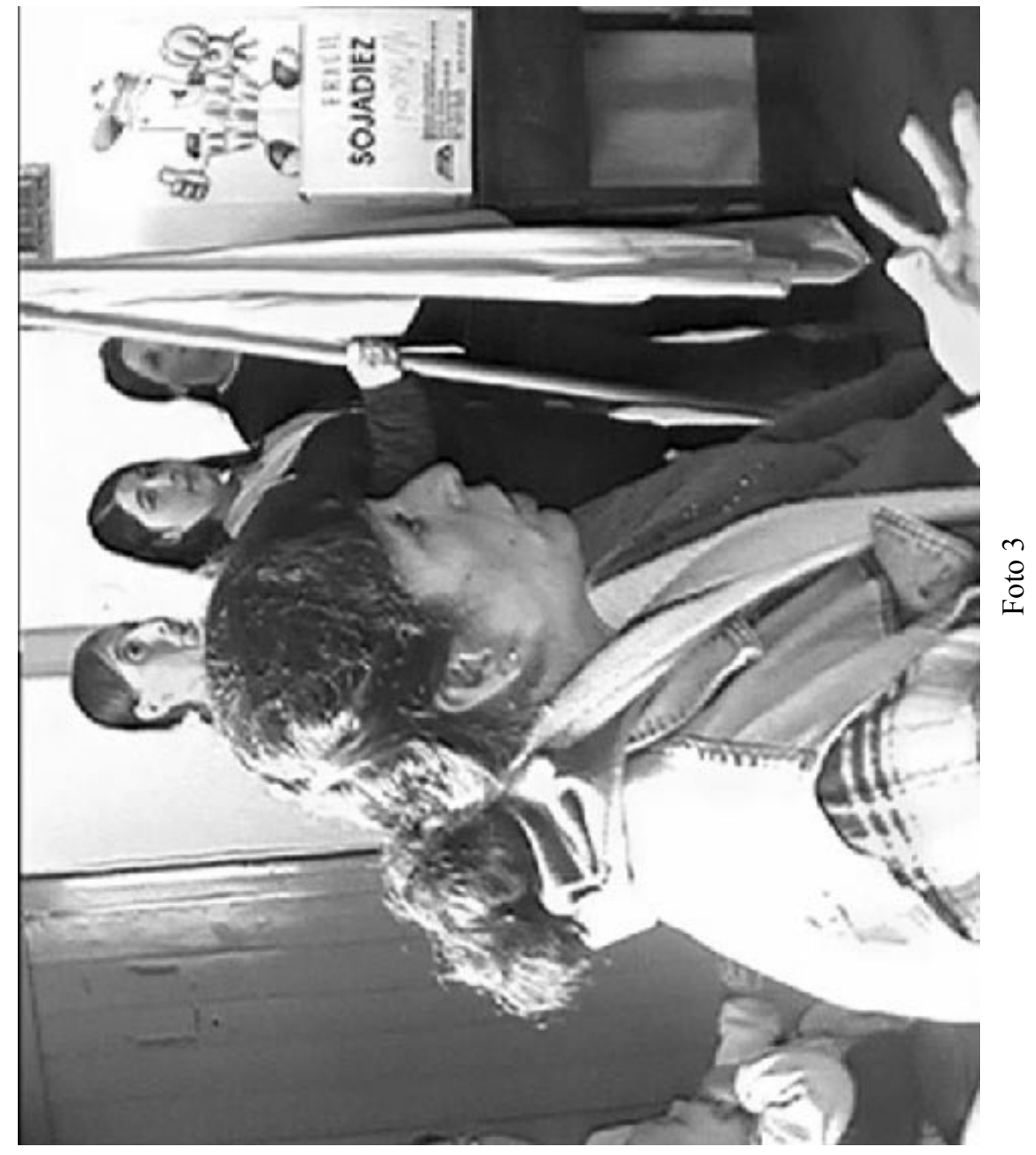





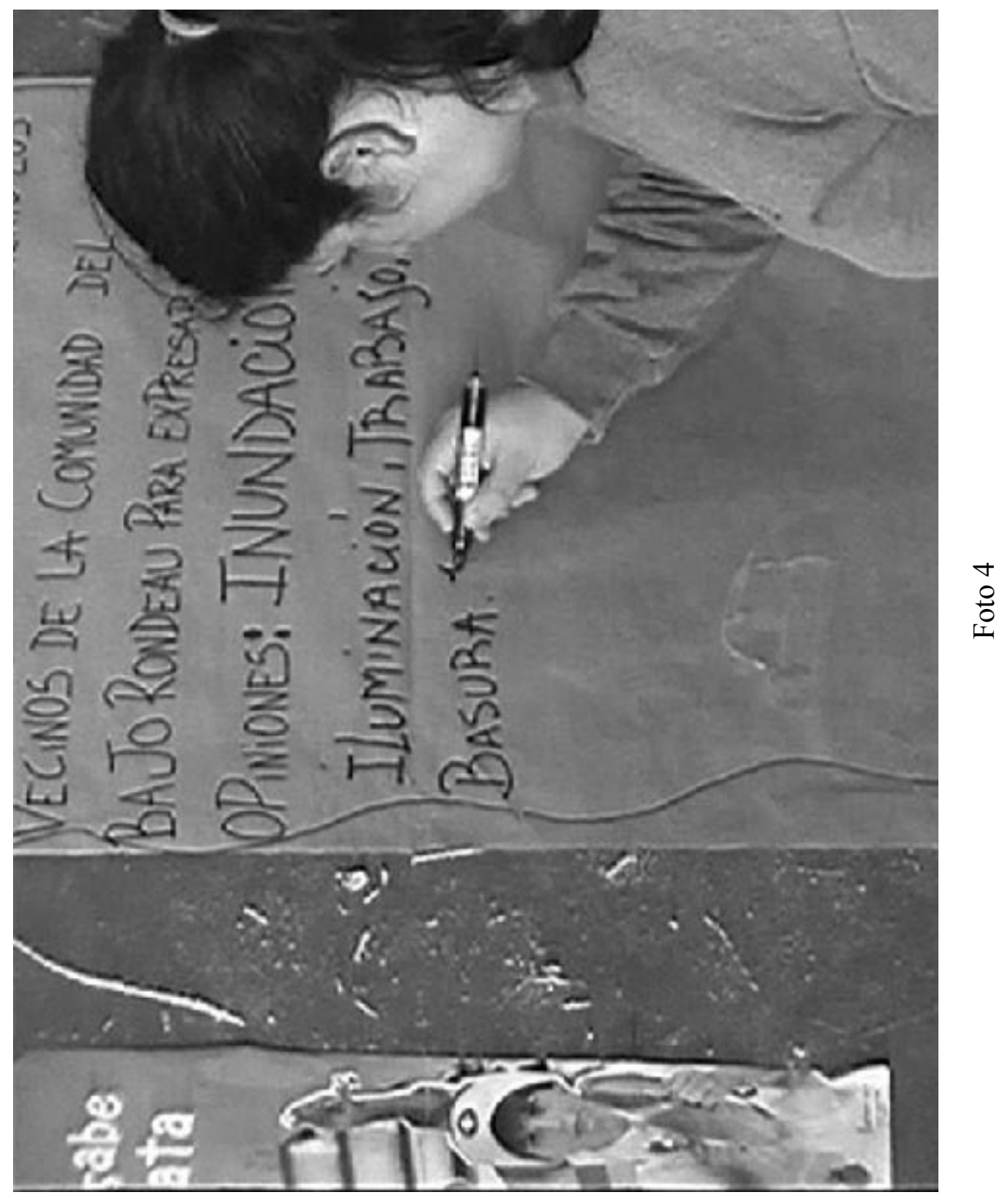


